\title{
Cisplatin and radiation therapy induces an immunologic clearance of HPV-positive head and neck cancer PW Nowicki1 ${ }^{1}$, WC Spanos ${ }^{3}$, DW Lee ${ }^{2}$, ME Anderson² ${ }^{2}$ AJ Hoover ${ }^{2}$ and JH Lee*3
}

\author{
Address: ${ }^{1}$ University of Iowa Dept. of Gyn/Onc, Iowa City, Iowa, USA, ${ }^{2}$ University of Iowa Dept of Otolaryngology, Iowa City, Iowa, USA and \\ ${ }^{3}$ Sanford Cancer Research Center, Sanford Health, Sioux Falls South Dakota, USA \\ * Corresponding author
}

\author{
from I Ith International Conference on Malignancies in AIDS and Other Acquired Immunodeficiencies (ICMAOI): Basic, Epidemiologic, and Clinical \\ Research \\ Bethesda, MD, USA. 6-7 October 2008
}

Published: 17 June 2009

Infectious Agents and Cancer 2009, 4(Suppl 2):OI6 doi:I0.1 I86/I750-9378-4-S2-OI6

This abstract is available from: http://www.infectagentscancer.com/content/4/S2/OI6

(c) 2009 Nowicki et al; licensee BioMed Central Ltd.

HPV is currently the most identifiable cause of head and neck squamous cell cancer (HNSCC). Intriguingly, although these tumors present at an advanced stage multiple studies have shown that they are more curable compared to HPV- HNSCC. To better understand why these more advanced tumors are curable, we have examined the response to treatment of HPV-positive cancer with radiation and cisplatin.

We have created a syngeneic mouse model of HPV-positive and HPV-negative HNSCC by transforming mouse primary tonsil epithelial cells with either HPV oncogenes or a non-antigenic RNAi strategy that affects similar oncogenic pathways. Using these transformed cells we examined the effect of radiation on HPV-positive and HPVnegative tumors in immune competent and immune incompetent mice. In addition to the mouse cells we also examined responses in human cancer cell lines. The results from our in vitro clonogenic survival assays demonstrate that HPV-positive cells are more resistant to radiation and cisplatin therapy compared to their HPVnegative counterparts. This result was consistent for human cancer cell lines, HPV transformed primary tonsil keratinocytes and HPV transformed mouse primary tonsil keratinocytes. Surprisingly the reverse sensitivity was observed in HPV-positive tumors after radiation and cisplatin therapy in vivo. HPV tumors were much more sensitive in vivo and at 20 gray of radiation HPV-positive tumors were eradicated compared to the HPV-negative counterpart that showed persistent growth. In the same manner, cisplatin therapy in vivo was able to result in a cure of HPV-positive tumor but not HPV-negative tumors. To understand whether an immune response could explain this enhanced eradication, we repeated the same studies in syngeneic mice lacking an ability to mount a cytolytic t-cell response. In these immune incompetent mice neither radiation nor cisplatin resulted in a cure. Adoptive transfer of wild-type immune cells into the immune incompetent mice restored immune clearance during cisplatin treatment. Our results prove that HPVpositive tumors are not more curable based on an increased epithelial sensitivity to cisplatin or radiation therapy, but rather that these therapies induce a tumor clearing immune response to this antigenic cancer. The implications from these results may lead to novel therapies that enhance tumor eradication for HPV-positive cancers. 